\title{
Nonalcoholic Wernicke Encephalopathy: An Entity Not to Be Missed!
}

\author{
Chee Chiat Liong, Kartini Rahmat, Jordina Siu Yi Mah, Shen-Yang Lim, Ai Huey Tan
}

Keywords: Wernicke, Encephalopathy, Ataxia, Coma, Nutrition

doi:10.1017/cjn.2016.269

Can J Neurol Sci. 2016; 43: 719-720

\section{Clinical Background}

A 52-year-old man was admitted for acute abdominal pain and vomiting. Laparotomy revealed dense adhesions, likely secondary to a ruptured appendix 30 years previously; adhesiolysis was performed and a segment of ileum resected. His postoperative course was complicated by recurrent bowel obstruction and persistent vomiting. Five weeks after his initial presentation, total parenteral nutrition was commenced. One day after this, he developed diplopia and bilateral horizontal nystagmus. There was no other medical history. He did not smoke or consume alcohol. Plain computed tomography of the brain was normal. Over the next several days, he developed an unsteady gait and gradual worsening of his conscious state (Glasgow Coma Scale score, E2V2M5 = 9). Tone was normal in all limbs and tendon reflexes were symmetrically brisk with flexor plantar responses. Limb power and sensory examination could not be tested reliably because of his poor conscious state. Eight days after the onset of neurological symptoms, he was intubated for airway protection. Serum electrolytes were normal. Brain magnetic resonance imaging (MRI) (Figure 1) showed T2 hyperintensities in the midbrain tectal plate/periaqueductal region, medial thalami, and mamillary bodies, findings that were highly suggestive of Wernicke encephalopathy (WE). ${ }^{1}$

\section{DISCUSSION}

WE is an acute neurologic complication resulting from thiamine (vitamin B1) deficiency. Thiamine replacement was started promptly, with clinical improvement. The patient was extubated on day 2 after treatment and was able to walk with a frame on day 7. Repeat brain MRI a week later showed near-complete resolution of the signal changes. After a month of treatment, he could walk independently, with minimal difficulty on tandem gait. The nystagmus has persisted after 1 year of treatment, but with no visual impairment.

A high index of suspicion for WE is needed in the setting of poor nutrition (e.g. caused by gastrointestinal disturbances, bariatric surgery, hyperemesis gravidarum, or malignancy), regardless of whether there is a history of alcoholism. ${ }^{1-4}$ WE can also be triggered by administration of total parenteral nutrition. ${ }^{4}$

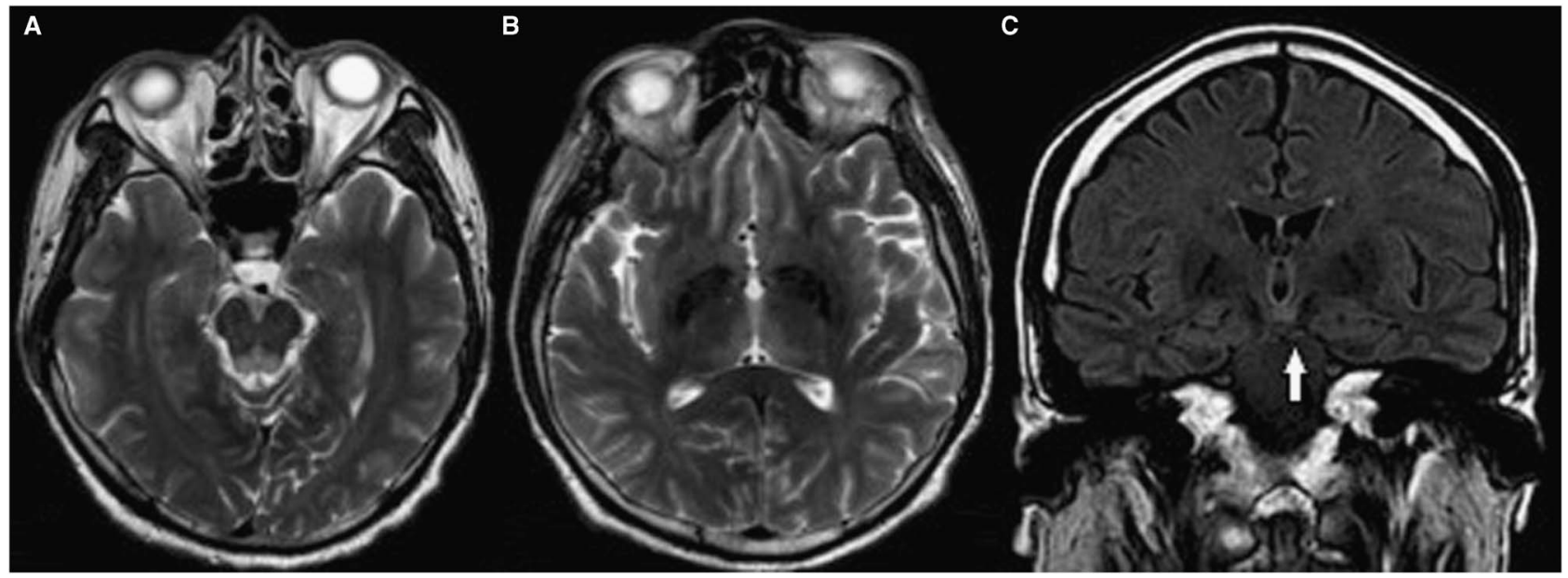

Figure 1: Typical MRI findings in Wernicke encephalopathy - Axial T2-weighted images showing hyperintensities in the periaqueductal area of the midbrain (A) and medial thalami (B); Coronal FLAIR image showing hyperintensities of the mamillary bodies $(C)$, as indicated by the arrow.

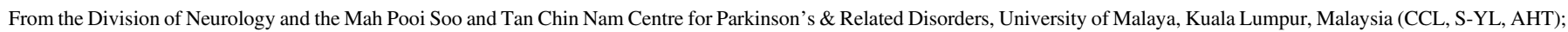
Department of Radiology, University of Malaya, Kuala Lumpur, Malaysia (KR); University of Glasgow Medical School, Glasgow, United Kingdom (JSYM)

Received November 24, 2015. Final Revisions Submitted May 9, 2016. Date of Acceptance May 21, 2016.

Correspondence to: Ai Huey Tan, Neurology Laboratory, Level 6, Menara Selatan (South Block), University of Malaya Medical Centre, 50603 Kuala Lumpur, Malaysia.

Email: aihuey.tan@gmail.com 


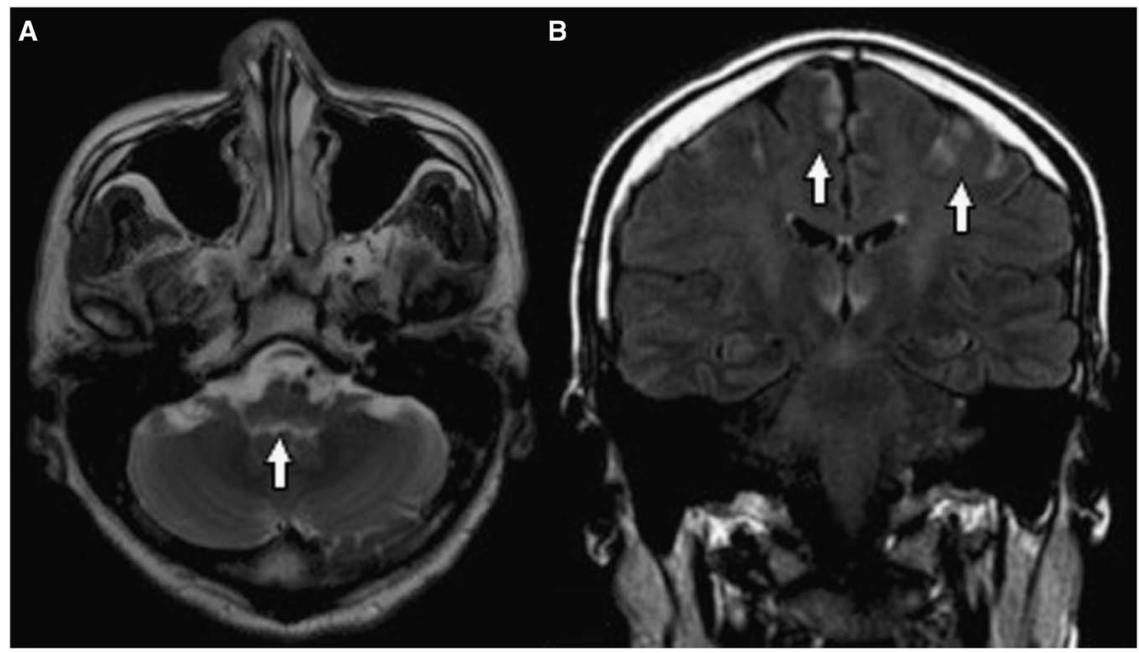

Figure 2: Atypical MRI findings in Wernicke encephalopathy - Axial T2-weighted image showing hyperintensities in dorsal medulla (A); Coronal FLAIR image showing cortical involvement (B).

In this case, it is likely that both the gastrointestinal disturbances and total parenteral nutrition were culprits. Often, not all features of the classic triad of ataxia, nystagmus/ophthalmoplegia, and confusion are seen, especially in nonalcoholic cases (the full triad occurring in only $33.6 \%$ of such cases versus $53.9 \%$ in alcoholic patients). ${ }^{2}$ Therefore, WE is often clinically underdiagnosed. ${ }^{1-4}$

Reversible cytotoxic edema in WE causes distinctive lesions and brain MRI is very useful in aiding the clinical diagnosis. ${ }^{1}$ The typical and common MRI findings of WE are symmetrical lesions in the medial thalami/periventricular region of the third ventricle (seen in $85 \%$ of cases), periaqueductal region $(65 \%$ of cases), and mamillary bodies (58\% of cases). The maintenance of cellular osmotic gradients in these brain regions has been postulated to be strictly related to thiamine concentration, thus rendering them vulnerable to thiamine deficiency. ${ }^{1}$ Interestingly, our patient's MRI also showed involvement of the dorsal medulla and cerebral cortex (Figure 2). In cases of nonalcoholic WE, atypical MRI findings can be observed in the cerebellum, cranial nerve nuclei, red nuclei, dorsal medulla, caudate nuclei, splenium, and cerebral cortex. ${ }^{1,3}$ Measurements of blood thiamine concentration or red blood cell transketolase activity are limited by a lack of specificity and technical difficulty, ${ }^{4}$ and were not done in this case.

The treatment of WE is immediate administration of parenteral thiamine. One recommended initial regime, instituted in our patient, is to give $500 \mathrm{mg}$ intravenously three times daily for 2 to 3 days; if a response is observed, this is followed by thiamine $250 \mathrm{mg}$ intravenously or intramuscularly daily for 3 to 5 days, or until clinical improvement ceases. ${ }^{4}$ Persistent neurological deficits such as impaired memory or learning disability, gait ataxia, and nystagmus are common. Early recognition and treatment of WE is critical to reduce the risk of permanent neurological sequelae or a fatal outcome. ${ }^{4}$

\section{Statement of Authorship}

The study concept was developed by AHT and SYL. Data were acquired by CCL, KR, JSYM, and AHT. The manuscript was drafted by CCL, AHT, SYL, KR, and JSYM.

\section{DisClOSURES}

None of the authors have anything to disclose.

\section{REFERENCES}

1. Zuccoli G, Gallucci M, Capellades J, et al. Wernicke encephalopathy: MR findings at clinical presentation in twenty-six alcoholic and nonalcoholic patients. Am J Neuroradiol. 2007;28:1328-31.

2. Galvin R, Brathen G, Ivashynka A, et al. EFNS guidelines for diagnosis, therapy and prevention of Wernicke encephalopathy. Eur J Neurol. 2010;17:1408-18.

3. Weidauer S, Nichtweiss M, Lanfermann H, Zanella FE. Wernicke encephalopathy: MR findings and clinical presentation. Eur Radiol. 2003;13:1001-9.

4. Sechi G, Serra A. Wernicke's encephalopathy: new clinical settings and recent advances in diagnosis and management. Lancet Neurol. 2007;6:442-55. 